\title{
On the meaning of Painlevé-Gullstrand synchronization
}

\author{
Xavier Jaén ${ }^{1 *}$ and Alfred Molina ${ }^{2 \dagger}$ \\ ${ }^{1}$ Dept. de Física i Enginyeria Nuclear, Universitat Politècnica de \\ Catalunya, Spain \\ ${ }^{2}$ Dept. Física Fonamental, Institut de Ciències del Cosmos, Uni- \\ versitat de Barcelona, Spain
}

May 7, 2015

\begin{abstract}
Following on from two recent papers, here we examine the relationship between Newtonian gravitation and general relativity in more depth. This allows us to define a scalar potential which is just the proper time of the vector potential when the latter is interpreted as the geodesic velocity field. The results are closely related to spacetimes that admit Painlevé-Gullstrand synchronization.
\end{abstract}

\section{Introduction}

The non-orthogonal coordinate system of Painlevé-Gullstrand [1, 2] is nowadays used to extend the Schwarzschild solution inside its event horizon. The Schwarzschild metric written in this coordinate system is regular across the horizon and is only singular for $r=0$. Another property that is very interesting is that its spatial geometry, the $t=$ constant surfaces, are flat; i.e. what is known as Painlevé-Gullstrand synchronization.

This type of synchronization is interesting in the context of gravitational collapse due to the fact that it allows us to go beyond the Schwarzschild radius. Such synchronization also has an increasing presence in the literature; for instance, in the so-called analog gravity models [3] or in relativistic hydrodynamics [4, where an effective Lorentzian metric is introduced.

*e-mail address: xavier.jaen@upc.edu

${ }^{\dagger}$ e-mail address: alfred.molina@ub.edu 
Our interest in metrics of this kind stems from the fact that they appear in a natural way when we generalize Newtonian mechanics to general relativity by means of a vector potential that includes gravity and inertial forces on the same footing [5]. In that earlier work we constructed Newtonian gravitation in which the field is derived from a vector potential $\vec{v}_{g}$ that can be interpreted as the velocity field of the trajectory solutions of the equations of motion. Furthermore, the field equations are invariant under the group of rigid motions. This generalization of Newtonian mechanics has a relativistic version, so a significant set of spacetimes can be written in a system of coordinates such that the metric is shape-invariant under the group of rigid motions. Among those spacetimes is the Schwarzschild solution written in Painlevé-Gullstrand coordinates [1]. Another interesting property of these spacetimes is that their non-relativistic limit can be obtained by making $c \rightarrow \infty$ without any consideration regarding weak fields.

In this paper we extend the previous type of metrics introduced in [5] to a more general one. Besides the vector potential, a scalar potential is introduced that is related to the proper time for the trajectories of free particles. To achieve this generalization, it is very helpful to consider Newtonian gravitation before studying the relativistic case. So first, we analyse Newtonian gravity in relation to the vector potential and the fact that it can be interpreted as a velocity field which is a solution of the equations of motion.

The paper is organized as we explain in what follows. In Sect. 2, we study the change produced in the Newtonian Lagrangian under different choices for the potential velocity field. In Sect. 3, we study the behavior of the relativistic Lagrangian when we change the potential velocity field. In the same section we state the problem and study Minkowski spacetime, introducing vector potentials, in order to express the metric, which are solutions of the equations of motion. As a consequence, we see that we must introduce a new scalar potential. In Sect. 4, we try to generalize the results of the previous section to a spacetime which depends on four potentials. We see how the properties of these spacetimes are inherited from Newtonian gravitation. In Sect. 5, we see the close relationship between the metrics studied and the family of metrics that support a Painlevé-Gullstrand synchronization [6, 7]. In Sect. 6, we study the form of the metrics under consideration when expressed in adapted coordinates. The results are compared with those obtained by other authors. Finally, in Sect. 7, we incorporate into this framework the FRWL spacetime previously considered in [8]. 


\section{Newtonian frame}

In [5] we prove that we can obtain the trajectories of particles in a Newtonian gravity field given by the vector potential $\vec{v}_{g}$, from the Lagrangian:

$$
L=\frac{1}{2} m\left(\dot{\vec{x}}-\vec{v}_{g}(\vec{x}, t)\right)^{2}
$$

These equations are covariant under rigid motion transformations, i.e. they are the same for inertial and non-inertial frames. If an observer $K$ has a system of orthonormal coordinates $\left\{t, \vec{x}=x^{i} \vec{e}_{i}\right\}$, and notices a gravitational field given by the Lagrangian given in (1), then observer $K^{\prime}$ with a system of coordinates that are also orthonormal $\left\{t^{\prime}, \vec{x}^{\prime}=x^{\prime i} \vec{e}_{i}^{\prime}\right\}$, related to $\{t, \vec{x}\}$ through the rigid transformation:

$$
t^{\prime}=t ; \vec{x}=\vec{X}(t)+\vec{x}^{\prime}=X^{i}(t) \vec{e}_{i}+x^{\prime i} \vec{e}_{i}^{\prime}=X^{i}(t) \vec{e}_{i}+x^{\prime k} R_{k}^{i}(t) \vec{e}_{i},
$$

where $R_{k}^{i}(t)$ is a orthogonal matrix, perceives the same gravitational field (except for inertial forces), i.e. it is produced for the same mass distribution, given by the Lagrangian in (11), where $\vec{x} \rightarrow \vec{x}^{\prime}$ and $\vec{v}_{g} \rightarrow \vec{v}_{g}^{\prime}$. The new vector potential $\vec{v}_{g}^{\prime}\left(\vec{x}^{\prime}, t\right)$ is related to the old one through:

$$
\vec{v}_{g}^{\prime}\left(\vec{x}^{\prime}, t\right)=\vec{v}_{g}(\vec{x}, t)-\vec{v}_{0}(\vec{x}, t)
$$

where $\vec{v}_{0}(\vec{x}, t)$ is the velocity vector field of the trajectories in (2), i.e.:

$$
\overrightarrow{v_{0}}(\vec{x}, t)=\dot{\vec{X}}(t)+\vec{\Omega}(t) \times(\vec{x}-\vec{X}(t))
$$

where:

$$
\vec{\Omega}(t) \equiv \frac{1}{2} \sum_{j} R_{j}^{k}(t) \dot{R}_{j}^{\ell}(t) \vec{e}_{k} \times \vec{e}_{\ell}
$$

It is a trivial and remarkable fact that from the Lagrangian in (1), given the vector potential $\vec{v}_{g}(\vec{x}, t)$, the trajectories that are a solution of $\dot{\vec{x}}(t)=$ $\vec{v}_{g}(\vec{x}(t), t)$ are a solution of the equations of motion, i.e. the vector potential which gives the gravitational field is one of the set of velocity fields which are solutions of the equations of motion of the gravitational field. This result strongly suggests that we can use as a vector potential any other vector potentials $\vec{v}_{g}^{*}(\vec{x}, t)$ that correspond to another set of trajectories that are also solutions of the equations of motion for the same gravitational field. For instance, for the exterior field of a spherical mass $M$ we have the vector potential:

$$
\vec{v}_{g}(\vec{x}, t)=\sqrt{\frac{2 M G}{r}} \hat{r}
$$


which is the velocity field for the radial trajectories in the gravitational field of the spherical mass $M$ with $v_{\infty}=0$. But what is more interesting is that every velocity field $\vec{v}_{g}^{*}(\vec{x}, t)$ corresponding to another set of trajectories that are solutions of the equations of motion with a potential (6) is a valid vector potential for the same gravitational field. In the following expression, for each allowed election of the constants $\bar{E}, \bar{\Gamma}, \bar{\alpha}_{\phi}$, we have a velocity field solution of the equations of motion

$$
\vec{v}_{g}^{*}(\vec{x}, t)= \pm \sqrt{\frac{2 M G}{r}+2 \bar{E}-\frac{\bar{\Gamma}^{2}}{r^{2}}} \hat{r} \pm \frac{1}{r} \sqrt{\bar{\Gamma}^{2}-\frac{\bar{\alpha}_{\phi}^{2}}{\sin ^{2} \theta}} \hat{\theta}+\frac{1}{r \sin \theta} \bar{\alpha}_{\phi} \hat{\phi}
$$

For instance, the vector potential (6) corresponds to the choice $\bar{E}=0, \bar{\Gamma}=$ $0, \bar{\alpha}_{\phi}=0$ in (7). Equation (7) is a complete family of the velocity fields when the particles move under the potential given by (6) $)$. It is easy to prove that the acceleration field $\vec{g}$ and the rotation field $\vec{\beta}$ (see [5]) are the same when we change the vector potential field $\vec{v}_{g}(6)$ for another $\vec{v}_{g}^{*}$ in the family given by (7). At the Newtonian level, these are a kind of gauge transformation. Let us consider this, from $L$ given in (11) the momentum is defined as:

$$
\vec{p} \equiv \frac{\partial L}{\partial \dot{\vec{x}}}=m\left(\dot{\vec{x}}-\vec{v}_{g}\right) \quad \dot{\vec{x}}=\vec{v}_{g}+\frac{\vec{p}}{m}
$$

and the Hamiltonian is:

$$
\mathcal{H} \equiv \vec{p} \cdot \dot{\vec{x}}-\bar{L}=\frac{\vec{p}^{2}}{2 m}+\vec{v}_{g} \cdot \vec{p}
$$

The Hamilton-Jacobi equation for the action $S$ is:

$$
\mathcal{H}(\vec{x}, \vec{p}=\vec{\nabla} S, t)+\frac{\partial S}{\partial t}=0
$$

or explicitly:

$$
\frac{1}{2 m}(\vec{\nabla} S)^{2}+\vec{v}_{g} \cdot \vec{\nabla} S+\frac{\partial S}{\partial t}=0
$$

If we have a solution $S$ of equation (11), then $\vec{p}=\vec{\nabla} S$ and from equation (8) or equivalently from $\dot{\vec{x}}=\frac{\partial \mathcal{H}}{\partial \vec{p}}$, we can construct the trajectory:

$$
\dot{\vec{x}}=\vec{v}_{g}+\frac{\vec{\nabla} S}{m}
$$

This equation suggests that we can define a new vector potential (gauge transformation) $\vec{v}_{g}^{*}=\vec{v}_{g}+\frac{\vec{\nabla} S}{m}$ which leads to the same gravitational and 
rotational fields, $\vec{g}$ and $\vec{\beta}$. The generator of this transformation is the action $S$.

We now show the relation between the Lagrangian $L$ in (1) and the new one $L^{*}$ :

$$
\begin{aligned}
L^{*}=\frac{m}{2}\left(\dot{\vec{x}}-\vec{v}_{g}^{*}\right)^{2} & =\frac{m}{2}\left(\dot{\vec{x}}-\left(\vec{v}_{g}+\frac{\vec{\nabla} S}{m}\right)\right)^{2}= \\
= & L+\frac{1}{2 m}(\vec{\nabla} S)^{2}-\left(\dot{\vec{x}}-\vec{v}_{g}\right) \cdot \vec{\nabla} S
\end{aligned}
$$

The second and third terms on the right-hand side of the last expression, taking into account the Hamilton-Jacobi equation (11), can be written as:

$$
\frac{1}{2 m}(\vec{\nabla} S)^{2}-\left(\dot{\vec{x}}-\vec{v}_{g}\right) \cdot \vec{\nabla} S=-\frac{d S}{d t}
$$

The difference between the two Lagrangians is the total derivative with respect to $t$ of a function, i.e. they are equivalent. The gauge transformations for the vector potential we have studied produce a change in the nonrelativistic Lagrangian which is a total derivative with respect to $t$ of the action; as is well known, this total time derivative does not change the Lagrange equations. This is why in Newtonian mechanics, we have the possibility of writing the field in terms of one vector potential or another; it does not matter. What is interesting is that the set of all possible trajectories of the particles is determined from knowledge of a single vector potential which is a particular velocity field of the particle trajectories.

\section{Relativistic frame}

In [5] we consider the relativistic extension of the Lagrangian in (10), that is:

$$
L=-m c^{2} \sqrt{1-\frac{\left(\dot{\vec{x}}-\vec{v}_{g}\right)^{2}}{c^{2}}},
$$

The particle trajectories are the geodesics of the spacetime metric:

$$
d s^{2}=-\left(c^{2}-\vec{v}_{g}^{2}\right) d t^{2}+d \vec{x}^{2}-2 \vec{v}_{g} \cdot d \vec{x} d t
$$

which has the following properties:

- it is shape-invariant under the group of rigid motions (2), provided that the local velocity field transforms as in (3). (Equation (14) is a particular case of the so-called Newtonian metrics [9].) 
- in the limit $c \rightarrow \infty$, it gives the Newtonian equations with no need for any weak-field approximation.

- The integrals of the vector potential $\vec{v}_{g}$, i.e. the solutions of $\dot{\vec{x}}=\vec{v}_{g}$, are geodesics.

One known example of this type of metric is the Schwarzschild metric written in Painlevé-Gullstrand coordinates. But it is clear that to describe all the possibilities in general relativity (where up to six potentials are needed) this type of metric based on three potentials is not enough. In [8] we add a new potential, $H_{g}(\vec{x}, t)$, to describe cosmological metrics such as the FRWL metric. At the end of this paper, in Sect. 7 below, we incorporate FRWL spacetimes.

The problem with metrics of the form given in equation (15) is that they are not invariant under the gauge transformations described at the Newtonian level, where the change of $\vec{v}_{g} \rightarrow \vec{v}_{g}^{*}$ only changes the Lagrangian by a total derivative of the action with respect to $t$. In the metric given in (15), when we substitute $\vec{v}_{g}$, given in equation (6) that gives the Schwarzschild metric written in Painlevé-Gullstrand coordinates, by $\vec{v}_{g}^{*}$, which should be the relativistic version of equation (7), the metric is not the Schwarzschild metric anymore.

Now we will try to identify the problem which appears in the relativistic scheme when we perform the relativistic version of Newtonian gauge transformations. To this end, we will begin by studying the easiest relativistic spacetime: the Minkowski metric. We write the Minkowski metric for an observer with orthonormal coordinates $\vec{x}$ and the time is given by watches that are at rest in the $\vec{x}$ points.

$$
d s^{2}=-c^{2} d \lambda^{2}+d \vec{x}^{2}
$$

$\lambda$ is the proper time for particles at rest. Let us now assume that the observer at the point $\vec{x}$ does not use the proper time at that point but would like to use the time of watches moving following another solution of the equations of motion, for instance a set of watches moving with constant velocity. As we will see, these solutions will give us a velocity field $\vec{v}_{g}^{*}(\vec{x}, \lambda)$ but also a proper time field $\tau_{g}^{*}(\vec{x}, \lambda)$.

From the Lagrangian associated with equation (16) $1,1, L=\sqrt{1-\frac{\dot{\vec{x}}^{2}}{c^{2}}}$ where $\dot{\vec{x}}=\frac{d \vec{x}}{d \lambda}$, we obtain the equation for the action $S$ :

$$
\partial_{\lambda} S=\sqrt{1+c^{2}(\vec{\nabla} S)^{2}}
$$

\footnotetext{
${ }^{1}$ From now on for simplicity we are going to miss out the $-m c^{2}$ factor in equation (14)
} 
Using the expression for the momentum and the inverse relation we have:

$$
\vec{p}=-\frac{\dot{\vec{x}}}{c^{2} \sqrt{1-\frac{\dot{\vec{x}}^{2}}{c^{2}}}}, \quad \dot{\vec{x}}=-\frac{c^{2}}{\sqrt{1+c^{2} p^{2}}} \vec{p}
$$

The velocity equation above suggests that we can construct a vector potential field $\vec{v}_{g}^{*}=\dot{\vec{x}}$ i.e.:

$$
\vec{v}_{g}^{*}=-\frac{c^{2}}{\sqrt{1+c^{2}(\vec{\nabla} S)^{2}}} \vec{\nabla} S
$$

Now we are going to relate the proper time to the action $S$. If $\vec{x}(\lambda)$ is a solution of the equation $\dot{\vec{x}}(\lambda)=\vec{v}_{g}^{*}(\vec{x}(\lambda), \lambda)$, then we are going to see that the proper time $\tau_{g}^{*}(\vec{x}, \lambda)$, is the action $S(\vec{x}, \lambda)$. Over the trajectories $\dot{\vec{x}}(\lambda)=$ $\vec{v}_{g}^{*}(\vec{x}(\lambda), \lambda)$ we have:

$$
d \tau_{g}^{*}=\sqrt{1-\frac{\vec{v}_{g}^{* 2}}{c^{2}}} d \lambda
$$

and also:

$$
d \tau_{g}^{*}=\left(\partial_{\lambda} \tau_{g}^{*}+\vec{v}_{g}^{*} \cdot \vec{\nabla} \tau_{g}^{*}\right) d \lambda
$$

and the equality on the trajectories of the second member of both equations:

$$
\partial_{\lambda} \tau_{g}^{*}+\vec{v}_{g}^{*} \cdot \vec{\nabla} \tau_{g}^{*}=\sqrt{1-\frac{\vec{v}_{g}^{* 2}}{c^{2}}}
$$

We are looking for a field $\tau_{g}^{*}$ that on the trajectories verifies equation (20). If we have a field that verifies equation (20) at every point $(\vec{x}, \lambda)$, then it would also verify equation (20) on the trajectories. We are going to consider equation (20) as an equation for $\tau_{g}^{*}(\vec{x}, \lambda)$ with $\vec{v}_{g}^{*}(\vec{x}, \lambda)$ known. Then, from equation (19), we can write equation (20) as:

$$
\partial_{\lambda} \tau_{g}^{*}=\frac{1+c^{2} \vec{\nabla} S \cdot \vec{\nabla} \tau_{g}^{*}}{\sqrt{1+c^{2}(\vec{\nabla} S)^{2}}}
$$

and comparing with the Hamilton-Jacobi equation (17), equation (21) has the solution for $\tau_{g}^{*}: \tau_{g}^{*}(\vec{x}, \lambda)=S(\vec{x}, \lambda)$. So the action $S$ solution of the HamiltonJacobi which generates the vector potential $\vec{v}_{g}^{*}(\vec{x}, \lambda)$ through equation (19) can be identified with the proper time of this velocity field. From now on, we identify $\tau_{g}^{*}$ and the action $S$. This identification has been proposed by other authors, related to a Gaussian coordinate system [10]. 
Now it is easy to prove that the metric in (16) can be written from $\vec{v}_{g}^{*}(\vec{x}, \lambda)$ and $\tau_{g}^{*}(\vec{x}, \lambda)$ as:

$$
d s^{2}=-c^{2} d \tau_{g}^{* 2}+\left(d \vec{x}-\vec{v}_{g}^{*} d \lambda\right)^{2}+c^{2}\left(\vec{\nabla} \tau_{g}^{*} \cdot\left(d \vec{x}-\vec{v}_{g}^{*} d \lambda\right)\right)^{2}
$$

i.e. taking into account equations (17) and (19), we can obtain $\partial_{\lambda} \tau_{g}^{*}$ and $\vec{v}_{g}^{*}$ :

$$
\partial_{\lambda} \tau_{g}^{*}=\sqrt{1+c^{2}\left(\vec{\nabla} \tau_{g}^{*}\right)^{2}}, \quad \vec{v}_{g}^{*}=-\frac{c^{2}}{\sqrt{1+c^{2}\left(\nabla \tau_{g}^{*}\right)^{2}}} \vec{\nabla} \tau_{g}^{*}
$$

and substituting them in equation (22), we obtain exactly the same expression (16).

It is important to note that the metric in (22) may have some ambiguity in the time coordinate used. We can use one of two time coordinates $\tau_{g}^{*}$ or $\lambda$. If we do not state otherwise, we will use $\lambda$. This means that $d \tau_{g}^{*}$ is a short way of writing $d \tau_{g}^{*}=\partial_{\lambda} \tau_{g}^{*} d \lambda+d \vec{x} \cdot \vec{\nabla} \tau_{g}^{*}$. It is also important to note that the change of gauge gives exactly the same metric, and therefore the same relativistic Lagrangian. This can be explained because the relativistic origin for the energy is fixed by the rest mass.

\section{Special potentials for general relativity}

We are going to construct a spacetime metric invariant for a class of observers in general relativity from a vector potential $\vec{v}_{g}(\vec{x}, \lambda)$ and a proper time $\tau_{g}(\vec{x}, \lambda)$. The metric constructed from these fields, following the previous Minkowski example, can be written as:

$$
d s^{2}=-c^{2} d \tau_{g}^{2}+\left(d \vec{x}-\vec{v}_{g} d \lambda\right)^{2}+c^{2}\left(\vec{\nabla} \tau_{g} \cdot\left(d \vec{x}-\vec{v}_{g} d \lambda\right)\right)^{2}
$$

Some interesting properties of this kind of metrics are:

- the metric is shape invariant under transformations where $\lambda$ remains fixed and $\vec{x}$ transforms as in (2), i.e. a rigid Newtonian transformation ( $\lambda$ represent a kind of absolute Newtonian time) if $\tau_{g}$ transforms as a scalar function, $\tau_{g}^{\prime}\left(\vec{x}^{\prime}, \lambda\right)=\tau_{g}(\vec{x}, \lambda)$, and $\vec{v}_{g}(\vec{x}, \lambda)$ transforms as in (3) as a Newtonian velocity field.

- if we assume that $\tau_{g}=\lambda+\frac{f(\vec{x}, \lambda)}{c^{2}}$, we obtain the Newtonian nonrelativistic limit simply by making $c \rightarrow \infty$, with no need for any weakfield approximation. 
- we can define the linear momentum as usual

$$
\vec{p} \equiv \frac{\partial L}{\partial \dot{\vec{x}}}=\frac{1}{L}\left(\left(\partial_{\lambda} \tau_{g}+\vec{v}_{g} \cdot \vec{\nabla} \tau_{g}\right) \vec{\nabla} \tau_{g}+\frac{1}{c^{2}}\left(\vec{v}_{g}-\dot{\vec{x}}\right)\right)
$$

then $\mathcal{H} \equiv \dot{\vec{x}} \cdot \vec{p}-L$ can be written as:

$$
\begin{aligned}
\mathcal{H}=\quad & \vec{v}_{g} \cdot \vec{p}+\left(\partial_{\lambda} \tau_{g}+\vec{v}_{g} \cdot \vec{\nabla} \tau_{g}\right) \\
& \left(c^{2} \vec{p} \cdot \vec{\nabla} \tau_{g}-\sqrt{\left(1+c^{2} \vec{p}^{2}\right)\left(1+c^{2}\left(\vec{\nabla} \tau_{g}\right)^{2}\right)}\right)
\end{aligned}
$$

and the Hamilton-Jacobi equation is:

$$
\partial_{\lambda} S(\vec{x}, \lambda)+\mathcal{H}(\vec{x}, \vec{p}=\vec{\nabla} S(\vec{x}, \lambda), \lambda)=0
$$

If we have a particular solution $S$ of this equation, we can obtain the equation of the trajectory:

$$
\begin{aligned}
& \dot{\vec{x}}=\left.\frac{\partial \mathcal{H}}{\partial \vec{p}}\right|_{\vec{p}=\vec{\nabla} S}= \\
& \vec{v}_{g}+c^{2}\left(\partial_{\lambda} \tau_{g}+\vec{v}_{g} \cdot \vec{\nabla} \tau_{g}\right)\left(\vec{\nabla} \tau_{g}-\frac{\left(1+c^{2}\left(\vec{\nabla} \tau_{g}\right)^{2}\right) \vec{\nabla} S}{\sqrt{\left(1+c^{2}(\vec{\nabla} S)^{2}\right)\left(1+c^{2}\left(\vec{\nabla} \tau_{g}\right)^{2}\right)}}\right)
\end{aligned}
$$

It is easy to see that a particular solution of the Hamilton-Jacobi equation is $S=\tau_{g}$ and for this solution we have

$$
\dot{\vec{x}}=\vec{v}_{g}
$$

Consequently, the trajectory solutions of $\dot{\vec{x}}(\lambda)=\vec{v}_{g}(\vec{x}(\lambda), \lambda)$ are solutions of the Lagrange equations i.e. they are geodesics of the metric in (24).

- It is invariant under the changes between members of the family of potentials representing different velocity fields and proper times of the corresponding family of geodesics for the metric in (24). That is, for each particular solution of the Hamilton-Jacobi equation $S$, we can take $\tau_{g}^{*}=S$ and $\vec{v}_{g}^{*}=\left.\frac{\partial \mathcal{H}}{\partial \vec{p}}\right|_{\vec{p}=\vec{\nabla} \tau_{g}^{*}}$. The metric expressed in these new potentials, $\tau_{g}^{*}$ and $\vec{v}_{g}^{*}$, is exactly the same as that in (24) and also is written in the same coordinate system. 


\section{Rigid coordinates and Painlevé-Gullstrand synchronization}

Metric (24) can be expressed in terms of the rigid Newtonian coordinates $\{\lambda, \vec{x}\}$ as:

$$
\begin{aligned}
d s^{2}= & -c^{2}\left(\frac{\partial \tau_{g}{ }^{2}}{\partial \lambda}-\left(\vec{\nabla} \tau_{g} \cdot \vec{v}_{g}\right)^{2}-\frac{\vec{v}_{g}^{2}}{c^{2}}\right) d \lambda^{2}- \\
& 2 c^{2}\left(\frac{\vec{v}_{g}}{c^{2}}+\left(\frac{\partial \tau_{g}}{\partial \lambda}+\vec{v}_{g} \cdot \vec{\nabla} \tau_{g}\right) \vec{\nabla} \tau_{g}\right) d \vec{x} d \lambda+d \vec{x}^{2}
\end{aligned}
$$

This belongs to a family of Newtonian metrics [9]:

$$
d s^{2}=-\Phi(\vec{x}, \lambda) d \lambda^{2}+2 \vec{K}(\vec{x}, \lambda) \cdot d \vec{x} d \lambda+d \vec{x}^{2}
$$

the interest in which stems from is based on the existence of the flat slicing $\lambda=$ constant [2]; that is, in our rigid Newtonian coordinate system, the spacetime exhibits Painlevé-Gullstrand synchronization.

Conversely, given a spacetime metric of the type (28), we can always construct the corresponding Hamilton-Jacobi equation:

$$
\partial_{\lambda} S=\vec{K} \cdot \vec{\nabla} S+\sqrt{\left(1+c^{2}(\vec{\nabla} S)^{2}\right)\left(\left(\frac{K}{c}\right)^{2}+\frac{\Phi}{c^{2}}\right)}
$$

This equation coincides with the scalar equation that we can build considering the equality between the metrics (27) and (28), by removing $\vec{v}_{g}$ and making $\tau_{g} \rightarrow S$.

For each particular solution for $S$, from (29) we obtain a scalar potential $S_{\text {particular }}=\tau_{g}$. The vector potential $\vec{v}_{g}$ can be obtained from the vector equation linking (27) and (28):

$$
\vec{v}_{g}=-\vec{K}-c^{2} \sqrt{\frac{\left(\frac{K}{c}\right)^{2}+\frac{\Phi}{c^{2}}}{1+c^{2}\left(\vec{\nabla} \tau_{g}\right)^{2}}} \quad \vec{\nabla} \tau_{g}
$$

Thus, we have demonstrated that given any spacetime that admits a Painlevé-Gullstrand synchronization, the Euclidean space coordinates are, in turn, rigid coordinates.

We have also found an interpretation of all Newtonian metrics in terms of a geodesic velocity field and its proper time. 


\section{Rigid vs. Painlevé-Novikov adapted to geodesic coordinates}

From expression (24), it is clear that the trajectory solutions of

$$
\frac{d \vec{x}}{d \lambda}=\vec{v}_{g}(\vec{x}(\lambda), \lambda)
$$

are geodesics of proper time $\tau_{g}$. The general solution of this equation can be expressed as $\vec{x}=\vec{\varphi}_{g}(\lambda, \vec{y})$, where $\vec{y}$ is the initial position, i.e. for $\lambda=\lambda_{0}$ we have $\vec{y}=\vec{\varphi}_{g}\left(\lambda_{0}, \vec{y}\right)$. The adapted geodesic coordinates $\{\lambda, \vec{y}\}$ can be defined through the relation $\vec{x}=\vec{\varphi}_{g}(\lambda, \vec{y})$. We have:

$$
d \vec{x}=\left(d \vec{y} \cdot \vec{\nabla}_{y}\right) \vec{\varphi}_{g}+d \lambda \partial_{\lambda} \vec{\varphi}_{g}
$$

but $\partial_{\lambda} \vec{\varphi}_{g}=\vec{v}_{g}\left(\vec{\varphi}_{g}, \lambda\right)$. Then we have:

$$
d \vec{x}-\vec{v}_{g}\left(\vec{\varphi}_{g}, \lambda\right) d \lambda=\left(d \vec{y} \cdot \vec{\nabla}_{y}\right) \vec{\varphi}_{g} \equiv \bar{d} \vec{\varphi}_{g}
$$

where $\bar{d} f \equiv\left(d \vec{y} \cdot \vec{\nabla}_{y}\right) f$ is the restriction of the differential form $d f$ to each slice, i.e. $d \lambda=0$.

Defining $\tilde{\tau}_{g}(\lambda, \vec{y}) \equiv \tau_{g}\left(\vec{x}=\vec{\varphi}_{g}(\lambda, \vec{y}), \lambda\right)$ we can write:

$$
\left.\vec{\nabla}_{x} \tau_{g}(\vec{x}, \lambda) \cdot\left(d \vec{x}-\vec{v}_{g}(\vec{x}, \lambda) d \lambda\right)\right|_{\vec{x}=\vec{\varphi}_{g}(\lambda, \vec{y})}=\bar{d} \tilde{\tau}_{g}(\lambda, \vec{y})
$$

By using the above relations, the metric in (24), in coordinates $\{\lambda, \vec{y}\}$, becomes:

$$
d s^{2}=-c^{2} d \tilde{\tau}_{g}^{2}+c^{2} \bar{d} \tilde{\tau}_{g}^{2}+\bar{d} \vec{\varphi}_{g}^{2}
$$

This metric is of the type:

$$
d s^{2}=-c^{2} d \tilde{\tau}_{g}^{2}+A_{i j}(\vec{y}, \lambda) d y^{i} d y^{j}
$$

which admit as geodesics $\vec{y}=$ constant with proper time $t=\tilde{\tau}_{g}$.

Furthermore, we can use the time coordinate $t$ adapted to the geodesic, i.e. the proper time of the geodesic $\vec{y}=$ constant, through the relation $t=\tilde{\tau}_{g}(\lambda, \vec{y})$

Given the potentials, $\tilde{\tau}_{g}$ and $\vec{\varphi}_{g}$, in terms of $(\vec{y}, \lambda)$, we can make the change of time $t=\tilde{\tau}_{g}(\vec{y}, \lambda) \leftrightarrow \lambda=\alpha_{g}(\vec{y}, t)$ which allows us to write metric (30) in the form:

$$
d s^{2}=-c^{2} d t^{2}+A_{i j} d y^{i} d y^{j}
$$

meaning that the coordinates $\{t, \vec{y}\}$ are Gaussian [10]. But it is important to note that not all Gaussian coordinates are of the type $\{t, \vec{y}\}$, i.e. adapted 
to a geodesic and rigid. Of course, not all spacetimes will admit a system of coordinates that are adapted to a geodesic and rigid; but all spacetimes admit Gaussian coordinates.

Using the potentials $\alpha_{g}(\vec{y}, t)$ and $\vec{\varphi}_{g}(\vec{y}, t) \equiv \vec{\varphi}_{g}\left(\lambda=\alpha_{g}(\vec{y}, t), \vec{y}\right)$ we can write the derivatives $\bar{d}$ in (30), which are restricted to $d \lambda=0$, as:

$$
\lambda=\alpha_{g}(\vec{y}, t) \Rightarrow d \lambda=d \vec{y} \cdot \vec{\nabla} \alpha_{g}+\partial_{t} \alpha_{g} d t=0 \Rightarrow \bar{d} t=-\frac{1}{\partial_{t} \alpha_{g}} d \vec{y} \cdot \vec{\nabla} \alpha_{g}
$$

then:

$$
\begin{gathered}
\bar{d} \tau_{g}=\bar{d} t=-\frac{1}{\partial_{t} \alpha_{g}} d \vec{y} \cdot \vec{\nabla} \alpha_{g} \\
\bar{d}_{\vec{\varphi}_{g}}=(d \vec{y} \cdot \vec{\nabla}) \overrightarrow{\breve{\varphi}}_{g}+\partial_{t} \overrightarrow{\breve{\varphi}}_{g} \bar{d} t=(d \vec{y} \cdot \vec{\nabla}) \overrightarrow{\breve{\varphi}}_{g}-\frac{1}{\partial_{t} \alpha_{g}}\left(d \vec{y} \cdot \vec{\nabla} \alpha_{g}\right) \partial_{t} \overrightarrow{\breve{\varphi}}_{g}
\end{gathered}
$$

The explicit form of (30) in adapted coordinates $\{t, \vec{y}\}$ is then:

$$
\begin{aligned}
d s^{2}= & -c^{2} d t^{2}+c^{2} \frac{1}{\left(\partial_{t} \alpha_{g}\right)^{2}}\left(d \vec{y} \cdot \vec{\nabla} \alpha_{g}\right)^{2}+ \\
& \left\{(d \vec{y} \cdot \vec{\nabla}) \vec{\varphi}_{g}-\frac{1}{\partial_{t} \alpha_{g}}\left(d \vec{y} \cdot \vec{\nabla} \alpha_{g}\right) \partial_{t} \vec{\varphi}_{g}\right\}^{2}
\end{aligned}
$$

Let us now study the special case of spherical symmetry. When we write the metric given in (24) in the rigid coordinate system $(r, \lambda)$, we have:

$$
\begin{aligned}
d s^{2}= & -c^{2} d \tau_{g}(r, \lambda)^{2}+\left(d r-v_{g}(r, \lambda) d \lambda\right)^{2}+ \\
& c^{2}\left(\partial_{r} \tau_{g}(r, \lambda)\left(d r-v_{g}(r, \lambda) d \lambda\right)\right)^{2}+r^{2} d \Omega^{2}
\end{aligned}
$$

The adapted spherical geodesic coordinates $\left\{\lambda, r_{0}\right\}$ are related to the previous rigid coordinates by:

$$
r=\varphi_{g}\left(\lambda, r_{0}\right), \quad \text { fulfilling } \quad \partial_{\lambda} \varphi_{g}=v_{g}\left(\varphi_{g}, \lambda\right)
$$

From (30) in the new coordinates $\left\{\lambda, r_{0}\right\}$, the metric is:

$$
d s^{2}=-c^{2} d \tilde{\tau}_{g}^{2}+c^{2} \bar{d} \tilde{\tau}_{g}^{2}+\bar{d} \varphi_{g}^{2}+\varphi_{g}^{2} d \Omega^{2}
$$

or more explicitly:

$$
\begin{aligned}
d s^{2}= & -c^{2}\left(\partial_{\lambda} \tilde{\tau}_{g} d \lambda+\partial_{r_{0}} \tilde{\tau}_{g} d r_{0}\right)^{2}+ \\
& \left(\left(\partial_{r_{0}} \varphi_{g}\right)^{2}+c^{2}\left(\partial_{r_{0}} \tilde{\tau}_{g}\right)^{2}\right) d r_{0}^{2}+\varphi_{g}^{2} d \Omega^{2}
\end{aligned}
$$


Following the same path that led us to (32), we can use the proper time $t$ of the geodesic $r_{0}=$ constant as the time coordinate, through the relation $t=\tilde{\tau}_{g}\left(\lambda, r=\varphi_{g}\left(\lambda, r_{0}\right)\right) \leftrightarrow \lambda=\alpha_{g}\left(r_{0}, t\right)$, together with $\breve{\varphi}_{g}\left(r_{0}, t\right)=\varphi_{g}(\lambda=$ $\left.\alpha_{g}\left(r_{0}, t\right), r_{0}\right)$. The explicit form of (30) in adapted spherical geodesic coordinates $\left\{\lambda, r_{0}\right\}$ is then:

$$
\begin{aligned}
d s^{2}= & -c^{2} d t^{2}+\left\{c^{2}\left(\frac{\partial_{r_{0}} \alpha_{g}}{\partial_{t} \alpha_{g}}\right)^{2}+\left(\frac{\partial_{r_{0}} \alpha_{g}}{\partial_{t} \alpha_{g}} \partial_{t} \breve{\varphi}_{g}-\partial_{r_{0}} \breve{\varphi}_{g}\right)^{2}\right\} d r_{0}^{2}+ \\
& \breve{\varphi}_{g}^{2} d \Omega^{2}
\end{aligned}
$$

\subsection{Schwarzschild}

If we substitute $\tau_{g}=\lambda$ and $\vec{v}_{g}=\sqrt{\frac{2 M G}{r}} \hat{r}$ in (24), we obtain the Schwarzschild metric written in Painlevé-Gullstrand coordinates [1, 5]. We can write this metric in adapted geodesic coordinates. We are going to use the geodesic radial trajectories with radial velocity zero at $r=\infty$ as the new radial coordinate:

$$
\tilde{\tau}_{g}(r, \lambda)=\lambda, \quad r=\varphi_{g}\left(\lambda, r_{0}\right)=\left(r_{0}^{3 / 2}+\frac{3}{2} \sqrt{2 M G}\left(\lambda-\lambda_{0}\right)\right)^{2 / 3}
$$

Note that in this case, the geodesic proper time $t=\tilde{\tau}_{g}$ coincides with $\lambda$. The metric written in these coordinates using the expressions (33) and (35) is:

$$
d s^{2}=-c^{2} d \lambda^{2}+\frac{r_{0}}{r} d r_{0}^{2}+r^{2} d \Omega^{2}
$$

where $r$ is given in (35) as a function of $r_{0}$ and $\lambda$. This expression, if we take $\lambda_{0}=0$ and $r_{0}=\left(-\frac{3}{2} \sqrt{2 M G} \chi\right)^{2 / 3}$, coincides with expression (11.12) of the Schwarzschild exterior solution given by Lemaittre [11], (in the expressions of Lemaître $G=1, c=1$ ).

The same coordinates are used by Novikov [12. The relation with our coordinates is $\lambda_{0}=0$ and $r_{0}=\left(\frac{3}{2} \xi\right)^{2 / 3}$. In expression (3.21), Novikov used $F=1$. To obtain our expression exactly, he would have needed to take $F=\sqrt{2 G M}$. 


\section{Homothetic motions and conformally flat synchronization}

Finally, we can incorporate the FRWL-type spacetime [8] by considering the metric depending on the potentials $\vec{v}_{g}(\vec{x}, \lambda), \tau_{g}(\vec{x}, \lambda)$ and $H_{g}(\vec{x}, \lambda)$,:

$$
d s^{2}=-c^{2} d \tau_{g}^{2}+c^{2}\left(\vec{\nabla} \tau_{g} \cdot\left(d \vec{x}-\vec{v}_{g} d \lambda\right)\right)^{2}+\frac{1}{H_{g}^{2}}\left(d \vec{x}-\vec{v}_{g} d \lambda\right)^{2}
$$

Repeating the same reasoning as in Sect. 4 step by step, where $H_{g}=1$, we conclude that:

- metric (36) is shape invariant under the group of homothetic motions $\left\{X^{i}(\lambda), R_{j}^{i}(\lambda), H(\lambda)\right\}$, which generalize the group of rigid motions $\left\{X^{i}(\lambda), R_{j}^{i}(\lambda)\right\}\left[8\right.$, if the vector potential $\vec{v}_{g}(\vec{x}, \lambda)$ and $H_{g}(\vec{x}, \lambda)$ transform as $\vec{v}_{g}^{\prime}\left(\vec{x}^{\prime}, \lambda\right)$ and $H_{g}^{\prime}\left(\vec{x}^{\prime}, \lambda\right)$ :

$$
\vec{v}_{g}^{\prime}\left(\vec{x}^{\prime}, \lambda\right)=\vec{v}_{g}(\vec{x}, \lambda)-\vec{v}_{0}(\vec{x}, \lambda) ; \quad H_{g}^{\prime}\left(\vec{x}^{\prime}, \lambda\right)=H(\lambda) H_{g}(\vec{x}, \lambda),
$$

where $\vec{v}_{0}$ is now defined, not as in (4), but according to:

$$
\vec{v}_{0}(\vec{x}, \lambda)=\vec{V}(\lambda)+\vec{\Omega}(\lambda) \times(\vec{x}-\vec{X}(\lambda))+\frac{\dot{H}(\lambda)}{H(\lambda)}(\vec{x}-\vec{X}(\lambda)),
$$

where $\vec{\Omega}$ is defined in (5) and " $\times$ " is the ordinary vector product; and $\tau_{g}$ transforms as a scalar.

- if $\tau_{g}=\lambda+\frac{f(\vec{x}, \lambda)}{c^{2}}$ and $H_{g}(\vec{x}, \lambda)=H_{g}(\lambda)+\frac{F(\vec{x}, \lambda)}{c^{2}}$, then we obtain the Newtonian non-relativistic limit simply by making $c \rightarrow \infty$, with no need for any weak-field approximation.

- we can define the linear momentum as usual:

$$
\vec{p} \equiv \frac{\partial L}{\partial \dot{\vec{x}}}=\frac{1}{L}\left(\left(\partial_{\lambda} \tau_{g}+\vec{v}_{g} \cdot \vec{\nabla} \tau_{g}\right) \vec{\nabla} \tau_{g}+\frac{1}{c^{2} H_{g}^{2}}\left(\vec{v}_{g}-\dot{\vec{x}}\right)\right)
$$

and then $\mathcal{H} \equiv \dot{\vec{x}} \cdot \vec{p}-L$ can be written as:

$$
\begin{aligned}
\mathcal{H}= & \vec{v}_{g} \cdot \vec{p}+\left(\partial_{\lambda} \tau_{g}+\vec{v}_{g} \cdot \vec{\nabla} \tau_{g}\right) \\
& \left(c^{2} H_{g}^{2} \vec{p} \cdot \vec{\nabla} \tau_{g}-\sqrt{\left(1+c^{2} H_{g}^{2} \vec{p}^{2}\right)\left(1+c^{2} H_{g}^{2}\left(\vec{\nabla} \tau_{g}\right)^{2}\right)}\right)
\end{aligned}
$$


and the Hamilton-Jacobi equation is:

$$
\partial_{\lambda} S(\vec{x}, \lambda)+\mathcal{H}(\vec{x}, \vec{p}=\vec{\nabla} S(\vec{x}, \lambda), \lambda)=0
$$

If we have a particular solution of this equation, we can obtain the trajectory equation:

$$
\begin{aligned}
\dot{\vec{x}}= & \left.\frac{\partial \mathcal{H}}{\partial \vec{p}}\right|_{\vec{p}=\vec{\nabla} S}=\vec{v}_{g}+c^{2} H_{g}^{2}\left(\partial_{\lambda} \tau_{g}+\vec{v}_{g} \cdot \vec{\nabla} \tau_{g}\right) \\
& \left(\vec{\nabla} \tau_{g}-\frac{\left(1+c^{2} H_{g}^{2}\left(\vec{\nabla} \tau_{g}\right)^{2}\right) \vec{\nabla} S}{\sqrt{\left(1+c^{2} H_{g}^{2}(\vec{\nabla} S)^{2}\right)\left(1+c^{2} H_{g}^{2}\left(\vec{\nabla} \tau_{g}\right)^{2}\right)}}\right)
\end{aligned}
$$

It is easy to see that a particular solution of the Hamilton-Jacobi equation is $S=\tau_{g}$ and for this solution we have:

$$
\dot{\vec{x}}=\vec{v}_{g}
$$

Consequently, the trajectory solutions of $\dot{\vec{x}}(\lambda)=\vec{v}_{g}(\vec{x}(\lambda), \lambda)$ are solutions of the Lagrange equations i.e. are geodesics of the metric in (36).

- It is invariant under the changes between members of the family of potentials, $\vec{v}_{g}$ and $\tau_{g}$, which represent different velocity fields and proper times of the corresponding family of geodesics for (36). That is, for each particular solution of the Hamilton-Jacobi equation $S$ we can take $\tau_{g}^{*}=S$ and $\vec{v}_{g}^{*}=\left.\frac{\partial \mathcal{H}}{\partial \vec{p}}\right|_{\vec{p}=\vec{\nabla} S}$. The metric related to these new potentials, $\tau_{g}^{*}$ and $\vec{v}_{g}^{*}$, now with $H_{g}$ unchanged, is exactly the same as in equation (36).

- The results of the slicing $\lambda=$ constant are now conformally flat $d s^{2}=$ $\frac{1}{H_{g}^{2}} d \vec{x}^{2}$. Metric (36) belongs to a family of metrics that admit a conformally flat synchronization

$$
d s^{2}=-\Phi(\vec{x}, \lambda) d \lambda^{2}+2 \vec{K}(\vec{x}, \lambda) \cdot d \vec{x} d \lambda+\frac{1}{H_{g}^{2}} d \vec{x}^{2}
$$

Conversely, given a spacetime metric of type (41), we can always construct the corresponding Hamilton-Jacobi equation: 


$$
\partial_{\lambda} S=H_{g}^{2} \vec{K} \cdot \vec{\nabla} S+\sqrt{\left(1+c^{2} H_{g}^{2}(\vec{\nabla} S)^{2}\right)\left(H_{g}^{2}\left(\frac{K}{c}\right)^{2}+\frac{\Phi}{c^{2}}\right)}
$$

This equation coincides with the scalar equation that we can construct considering the equality between the metrics (36) and (41), by removing $\vec{v}_{g}$ and making $\tau_{g} \rightarrow S$.

For each particular solution $S$ of equation (42), we obtain a scalar potential $\tau_{g}=S$. The vector potential $\vec{v}_{g}$ can be obtained from the vector equation linking (36) and (41) or directly from the Hamiltonian.

$$
\vec{v}_{g}=-H_{g}^{2}\left(\vec{K}+c^{2} \sqrt{\frac{H_{g}^{2}\left(\frac{K}{c}\right)^{2}+\frac{\Phi}{c^{2}}}{1+c^{2} H_{g}^{2}\left(\vec{\nabla} \tau_{g}\right)^{2}}} \vec{\nabla} \tau_{g}\right)
$$

Thus, we have demonstrated that given any spacetime that supports a conformally flat synchronization, its conformal Euclidean coordinates are, in turn, homothetic coordinates.

\section{Conclusions}

This work is a continuation of two previous papers. In that previous work, we analysed some almost unknown properties of Newtonian gravity. We then tried to translated those properties to relativistic gravity. This approach allowed us, in the first paper, to provide a meaning for a family of metrics, which are nothing more than an extension of the Painlevé-Gullstrand type of metrics. In the second paper we were able to incorporate cosmological spacetimes, of the FRWL type, into a framework that can be considered a cosmological extension of the Painlevé-Gullstrand type of metrics.

In this way, we were able to find up to four potentials, which have their own meaning, from which to express a significant set of spacetime metrics.

It is clear that the set of spacetimes covered by the metrics we had found, with only four potentials, was not enough to include the set covered by standard general relativity. But the fact that the study of Newtonian gravity was so successful encouraged us to extend our research even further in the same direction.

To this end, in the present paper, we develop a new unknown property of Newtonian gravity; a property that already appeared as a curiosity in our 
previous work: the vector potential can be interpreted as a velocity field whose integral trajectories are solutions of the equations of motion.

This fact strongly suggests that the vector potential can be substituted by any other vector potential that is also a solution of the equations of motion. And that is indeed the case at the non-relativistic level. The field equations are invariant under this kind of gauge transformation.

The main contribution of this paper is to extend this property to the maximum number of spacetimes within standard general relativity. We clearly laid out the problem and we saw that we needed a new scalar potential whose meaning is the proper time of the vector potential. Studying the Minkowski metric allowed us to construct a sufficiently general family of metrics which inherit the properties of Newtonian gravity above mentioned.

The family of metrics found, expressed in a rigid coordinate system, is the same as the family of metrics with flat synchronization, i.e. those that exhibit Painlevé-Gullstrand synchronization.

Finally, we incorporated the cosmological spacetime into the general framework, without losing any of the properties inherited from Newtonian gravity. Now the family of metrics, expressed in homothetic coordinates, is the same as the family of metrics with conformally flat synchronization.

As a result, we obtain a family of spacetimes which support a conformally flat synchronization; the metric can be written via five potentials:

- Three components of the vector potential $\vec{v}_{g}$, plus one of the scalar potential $\tau_{g}$, from which we can form a geodesic four-vector of the metric which in turn it represents.

- A scalar potential $H_{g}$ which has the meaning of a local homothety.

All these potentials have some physical meaning, and even in Newtonian theory they leave a trace.

As is well known, the general theory of relativity is described by six potentials. In our approach, in which we have been guided by Newtonian theory, we have identified five of these six potentials. An interesting question is: is it possible to introduce the sixth potential without sacrificing the properties studied in this work? Then, if this is not possible: what are the properties we should dispense with?

The spacetimes covered in this work are identified with those that admit a flat or conformally flat synchronization. These spacetimes do not support gravitational waves. It seems that the introduction of the sixth potential will allow gravitational waves. Following these comments, it seems clear that the introduction of the sixth potential cannot arise from any previous study of Newtonian gravitation or of special relativity. The sixth potential may be 
most genuinely linked to general relativity, without leaving any trace possible of being included in Newtonian theory by some limiting process.

\section{Acknowledgments}

We thank Bartolomé Coll for suggestions we received at an early stage of the work; Juan Antonio Morales-Lladosa and Eduardo Ruiz for carefully reading a previous draft of the paper and providing useful criticism that led to improvements; and finally Lluís Bel, without whose inspiration and encouragement, hardly any of this series of papers would have occurred to us.

\section{References}

[1] Painlevé, P. C.R. Acad. Sci. (Paris)173, 677-680 (1921). Gullstrand, A. Ark. Mat. Astron. Fys., 16 (8), 1-15 (1922).

[2] Herrero, A. and Morales,J.A. Class. Quantum. Grav. 27 175007, (2010)

[3] Barceló, C., Liberati, S. and Visser, M. Living Rev. Relativity 14 3, (2011). DOI 10.12942/lrr-2011-3.

[4] Lichnerovicz, A. Relativistic Hydrodynamics and Magnetohydrodynamics Benjamin, (1967).

[5] Jaén, X. and Molina, A. Gen. Relativ. Gravit.45, 1531-1546, (2013) DOI $10.1007_{s} 10714-013-1542-9$.

[6] Bini, D. Geralico, A. and Jantzen, R.T. Gen. Relativ. Gravit.44, 603621, (2012) DOI 10.1007s10714-011-1295-2.

[7] Maulik K. Parikh, Physics Letters B 546 189-195 (2002).

[8] Jaén, X. and Molina, A. Gen. Relativ. Gravit.46, 1745, (2014) DOI $10.1007_{s} 10714-014-1745-8$.

[9] Bel, Ll. Recent developments in gravitation Ed. Verdaguer, Garriga, Cespedes World Scientific (1990).

[10] Novello, M. and Bittencourt, E. Gravitation and Cosmology 17 (3), 230241 (2011). 
[11] Lemaître, G. Annales de la Société Scientifique de Bruxelles A53 51-85 1993. Reproduced in Gen. Relativ. Gravit.29, 641-680 (1997).

[12] Novikov, I. D. Communications of the Shternberg State Astronomical Institute 132 3-42 (1964). Reproduced in Gen. Relativ. Gravit. 33 22592295 (2001). 